\title{
Variation Principles for an Arbitrary Operator. III
}

\author{
By L. M. Delves
}

\begin{abstract}
The methods, described in two previous papers, for generating variation principles for the matrix elements of Hermitian operators are extended here in several ways. The method is first extended to cover inhomogeneous equations. A defect of the original formulation, that it involved two trial functions, is removed by rewriting the principle so that one only appears. Finally, variation-iteration schemes are proposed.
\end{abstract}

1. Introduction. In two previous papers [1], [2], hereafter called I and II, the following problem was considered. Given two states $|\alpha\rangle,|\beta\rangle$, which are solutions of the homogeneous equations

$$
\begin{aligned}
& \left(H_{\alpha}-E_{\alpha}\right)|\alpha\rangle=0, \\
& \left(H_{\beta}-E_{\beta}\right)|\beta\rangle=0,
\end{aligned}
$$

find a variation principle for the matrix element $\langle\alpha|W| \beta\rangle$ of an arbitrary Hermitian operator $W$. We here extend these results in several ways. We first extend the method to cover inhomogeneous equations of the type $A|\gamma\rangle=|b\rangle$, where $|b\rangle$ is a given vector, and derive variation principles for the number $\langle\gamma|W| \gamma\rangle$.

Second, we are able to simplify the results somewhat. The principles derived involve two trial functions for each state $|\alpha\rangle$; in Section 3 we show that an alternative principle may be given involving a single trial function. This principle requires the inversion of the operator $W-E_{1}$, where $E_{1}$ is a constant; for many operators $W$ of practical interest, this inversion is trivial.

Finally, in Section 4 we give a suitable variation-iteration scheme for use with these principles, and thus a practical way of improving the results.

We use throughout the notation of finite matrices. Thus, the states $|\alpha\rangle,|\beta\rangle$ will be written $\alpha, \beta$; and the matrix element $\langle\alpha|W| \beta\rangle$ will be written $\alpha^{+} W \beta$. This notation is chosen for its brevity and ease of printing; the results are directly applicable also to differential operators $H_{\alpha}$, providing the boundary conditions are such that these are Hermitian. This requirement precludes scattering states from our discussion; the modifications to the method required to include these were given in II, and similar modifications go through for the cases treated here.

2. Inhomogeneous Equations. We consider a system represented by the state vector $x_{0}$ satisfying the (linear) inhomogeneous equation

$$
H x_{0}=b,
$$

where the matrix $H$ is hermitian; suppose that we wish to find a variation principle for the quantity $\langle W\rangle=x_{0}{ }^{+} W x_{0}$, for some Hermitian operator $W$. Then, following the procedures of $I$ and II, we consider the associated equation

$$
(H+\lambda W) x_{\lambda}=b,
$$

Received June 1, 1963. 
which leads to

$$
\begin{aligned}
H x_{1} & =-W x_{0}, \\
x_{1} & =\left.\frac{d x_{\lambda}}{d \lambda}\right|_{\lambda=0} .
\end{aligned}
$$

Then it is easy to show, following again the arguments of $I$, that a variational estimate of $\langle W\rangle$ is $[W]$, where

$$
[W]=x_{0}{ }^{+} H x_{1}+x_{1}{ }^{+} H x_{0}-b^{+} x_{1}-x_{1}{ }^{+} b+x_{0}{ }^{+} W x_{0} \text {. }
$$

Similarly for off-diagonal matrix elements: given two systems $x_{0}, y_{0}$ :

$$
\begin{aligned}
& H_{1} x_{0}=b, \\
& H_{2} y_{0}=c,
\end{aligned}
$$

we can define $x_{1}, y_{1}$ through

$$
\begin{aligned}
& H_{1} x_{1}=-W y_{0}, \\
& H_{2} y_{1}=-W x_{0},
\end{aligned}
$$

and find as a variation principle for $y_{0}{ }^{+} W x_{0}$ :

$$
\begin{aligned}
\delta\left[y_{0}{ }^{+} W x_{0}\right] & =0, \text { where } \\
{\left[y_{0}{ }^{+} W x_{0}\right] } & =y_{0}{ }^{+} W x_{0}+y_{0}{ }^{+} H_{2} y_{1}+x_{1}{ }^{+} H_{1} x_{0}-c^{+} y_{1}-x_{1}{ }^{+} b .
\end{aligned}
$$

A variation principle for $x_{0}{ }^{+} W y_{0}$ follows from taking the conjugate transpose of (7).

An Example. As a simple sample of the use of these principles, we consider a set of linear equations (2) with

$$
\begin{aligned}
H & =\left(\begin{array}{ccc}
3 & 2 & 1 \\
2 & 1 & -1 \\
1 & -1 & 2
\end{array}\right), \\
b^{+} & =(10,1,5),
\end{aligned}
$$

and suppose we are interested in the number $\sum x_{0 i}^{2}=x_{0}{ }^{+} W_{1} x_{0}=\left\langle W_{1}\right\rangle$, where the operator $W_{1}=I$, the unit operator. The exact solution to (2) and (3) in this case is

$$
x_{0}^{+}=(1,2,3) ; \quad x_{1}^{+}=\left(-\frac{9}{5}, 2, \frac{2}{5}\right), \quad\left\langle W_{1}\right\rangle=14,
$$

and we assume we have found approximate solution $x_{0 t}, x_{1 t}$ :

$$
\begin{aligned}
& x_{0 t}^{+}=(0.9,2.2,3.3), \\
& x_{1 t}^{+}=(-1.9,1.9,0.5) .
\end{aligned}
$$

The errors in these approximate solutions are about $10 \%$. The vector $x_{0 t}$ gives, as a first approximation to $\left\langle W_{1}\right\rangle$ :

$$
\left\langle W_{1}\right\rangle_{t}=x_{0 t}^{+} W_{1} x_{0 t}=16.54,
$$

while the variational principle (4) gives, with this $x_{0 t}, x_{1 t}$ : 


$$
\left[W_{1}\right]=14.18
$$

with an error of about $1.3 \%$ compared to $18 \%$ in $\left\langle W_{1}\right\rangle_{t}$.

This is likely to be a particularly favourable example because of the regular form of $W$ chosen. However, we can repeat the calculation for a singular $W_{2}$. We choose

$$
W_{2}=\left(\begin{array}{lll}
0 & & \\
& 1 & \\
& & 0
\end{array}\right)
$$

for which $x_{1}^{+}=(-1,1,1)$ and $\left\langle W_{2}\right\rangle \equiv x_{02}^{2}=4$, so that the variation principle for this choice of $W$ gives an approximation to a single component of $x_{0}$.

With the same approximation as before for $x_{0 t}$ and $x_{1 t}^{+}=(-0.9,0.9,1.1)$, we find $\left\langle W_{2}\right\rangle_{t}=4.84,\left[W_{2}\right]=4.24$.

The improvement in this case, although marked, is less dramatic.

3. An Alternative Principle. The variation principles developed so far in these papers have required the use of two trial functions, $x_{0 t}$ and $x_{1 t}$, for each state $x_{0}$ of interest. It is possible to rewrite the equations so that only one of these functions, $x_{1}$, appears, while retaining the variational character of the results, and we do this here. The new principle then involves the operator $\left(E_{1}-W\right)^{-1}$; but when this operator can be evaluated, the new form may be easier to use than the old.

We consider here the eigenvalue equation treated in I. Suppose, for Hermitian $H$ and eigenvalue $E_{0}$,

$$
\left(H-E_{0}\right) x_{0}=0, \quad x_{0}{ }^{+} x_{0}=1,
$$

and that we are interested in

$$
\langle W\rangle=x_{0}{ }^{+} W x_{0} .
$$

We shall assume $E_{0}$ is known (as discussed in I, a variational approximation to $E_{0}$ is sufficient to retain the variational character of our results), and we shall write $H-E_{0}=L$. Then we define $x_{1}$ as a solution of the equation

$$
L x_{1}=\left(E_{1}-W\right) x_{0},
$$

so that

$$
E_{1}=x_{0}{ }^{+} L x_{1}+x_{0}{ }^{+} W x_{0}=\langle W\rangle .
$$

Then $x_{1}$ also satisfies the equation

$$
\begin{aligned}
A x_{1} & =0, \\
A & =L\left(E_{1}-W\right)^{-1} L .
\end{aligned}
$$

The operator $A$ is Hermitian, since $L$ and $W$ are Hermitian.

Then it is easy to show that a variation principle for $\langle W\rangle$ is $[W]$, where

$$
\begin{aligned}
{[W] } & =E_{1}+x_{1}{ }^{+} A x_{1}, \\
\delta[W] & =0 .
\end{aligned}
$$

This principle does not involve $x_{0}$ explicitly; methods of using (14) in practice are 
similar to those discussed in I. It may appear that an approximation $x_{0 t}$ to $x_{0}$ is still necessary, first to give an approximation $E_{1 t}$ to $E_{1}$ through

$$
E_{1 t}=x_{0 t}^{+} W x_{0}
$$

and second, to determine the normalization of $x_{1}$ through (12). This is not so, however; for a given form of $x_{1}$, which may itself contain a number of parameters, [W] is a function of $E_{1}$; the best value of $E_{1}$ is then that for which $\partial[W] / \partial E_{1}=0$.

Further, the normalization of $x_{1}$ may be specified by imposing the condition

$$
x_{1}^{+} L\left(E_{1}-W\right)^{-2} L x_{1}=1
$$

which, from (12), is equivalent to $x_{0}{ }^{+} x_{0}=1$, but which does not require a trial function for $x_{0}$.

The same procedure can be carried through for off-diagonal elements of $W$, but the results are unfortunately much more complicated. We assume we have

$$
\begin{array}{ll}
L_{1} x_{0}=0, & x_{0}{ }^{+} x_{0}=1, \\
L_{2} y_{0}=0, & y_{0}{ }^{+} y_{0}=1,
\end{array}
$$

for Hermitian $L_{1}, L_{2}$; and we are interested in $y_{0}{ }^{+} W x_{0}$ for some operator $W$.

We shall assume

$$
y_{0}{ }^{+} W x_{0}=x_{0}{ }^{+} W y_{0}
$$

so that the matrix element is real. Then we introduce functions $x_{1}, y_{1}$ satisfying

$$
\begin{aligned}
& L_{1} x_{1}=E_{1} x_{0}-W y_{0}, \\
& L_{2} y_{1}=E_{2} y_{0}-W x_{0},
\end{aligned}
$$

for which $E_{1}=x_{0}{ }^{+} W y_{0}, E_{2}=y_{0}{ }^{+} W x_{0}=E_{1}$ from condition (16). Then it is possible to show that a variation principle for $y_{0}{ }^{+} W x_{0}$ is

$$
\begin{aligned}
\delta\left[y_{1} W x_{0}\right] & =0 \\
\left.y_{0}{ }^{+} W x_{0}\right] & =E_{1}-\frac{1}{2}\left[x_{1}^{+} L_{1} E_{1} B L_{1} x_{1}+y_{1}{ }^{+} L_{2} E_{1} B L_{2} y_{1}\right. \\
& \left.+x_{1}^{+} L_{1} W B L_{2} y_{1}+y_{1}{ }^{+} L_{2} W B L_{1} x_{1}\right] \\
B & =\left(W^{2}-E_{1}^{2}\right)^{-1} .
\end{aligned}
$$

Equation (18) reduces to equation (14) when $L_{1}=L_{2}$.

As for the diagonal elements, it is necessary to fix the normalization of $x_{1}, y_{1}$ in some way. One possible way, which does not involve a knowledge of a trial function for $x_{0}, y_{0}$, is through the relations

$$
\begin{aligned}
& y_{1}{ }^{+} L_{2} E_{1} B^{2} W L_{1} x_{1}+y_{1}{ }^{+} L_{2} E_{1}{ }^{2} B^{2} L_{2} y_{1} \\
& +x_{1}{ }^{+} L_{1} W E_{1} B^{2} L_{2} y_{1}+x_{1}{ }^{+} L_{1} W^{2} B^{2} L_{1} x_{1}=1 \\
& x_{1}{ }^{+} L_{1} E_{1} B^{2} W L_{2} y_{1}+x_{1}{ }^{+} L_{1} E_{1}{ }^{2} B^{2} L_{1} x_{1} \\
& +y_{1}{ }^{+} L_{2} W E_{1} B^{2} L_{1} x_{1}+y_{1}{ }^{+} L_{2} W^{2} B^{2} L_{2} y_{1}=1
\end{aligned}
$$

Equations (19a), (19b) reduce to the conditions $x_{0}{ }^{+} x_{0}=1, y_{0}{ }^{+} y_{0}=1$, for exact solutions $x_{1}, y_{1}$ of $(17)$. 
It is interesting that together they imply

$$
x_{1}{ }^{+} L_{1} B L_{1} x_{1}=y_{1}{ }^{+} L_{2} B L_{2} y_{1},
$$

which may be used to simplify (18), since $E_{1}$ is a constant.

The complicated nature of (18) and (19) would seem to rule them out as a practical means of calculation; the original principle of II would appear easier to use.

Inhomogeneous Equations. The method carries through also for inhomogeneous equations of the type considered in Section 2 . We give here only the results for the diagonal elements. With the same notation as Section 2, a variation principle for $\langle W\rangle$ is

$$
[W]=-\left(x_{1}^{+} b+b^{+} x_{1}+x_{1}{ }^{+} H W^{-1} H x_{1}\right),
$$

for which $\delta[W]=0$, where $x_{1}$ satisfies (3) and (2).

An Example. If we repeat the example of Section 2 for $W=W_{1}=I$, and with the same approximation $x_{1 t}$ to $x_{1}$, we find, from (20),

$$
\left[W_{1}\right]=13.64 \text {. }
$$

Thus in this case, equation (20) gives less accurate results than equation (7), for the trial functions considered. Equation (20) cannot be used to calculate $\left\langle W_{2}\right\rangle$, since $W_{2}$ has no inverse.

4. A Variation-Iteration Scheme. So far we have assumed that approximations $x_{0 t}$ and $x_{1 t}$ to $x_{0}$ and $x_{1}$ are available. We give here one method by which a sequence of such approximations may be generated. For simplicity, we again limit the discussion to diagonal matrix elements of $W$, although off-diagonal elements may be considered similarly.

We consider the equations

$$
\begin{aligned}
& \left(H-E_{0}\right) x_{0}=0, \quad x_{0}{ }^{+} x_{0}=1, \\
& \left(H-E_{0}\right) x_{1}=\left(E_{1}-W\right) x_{0}, \quad E_{1}=x_{0}{ }^{+} W x_{0},
\end{aligned}
$$

and suppose that we have approximations $x_{1}{ }^{(n)}, E_{1}{ }^{(n)}, x_{0}{ }^{(n)}, E_{0}{ }^{(n)}$ to $x_{1}, E_{1}, x_{0}, E_{0}$.

Then we can define $(n+1)$ st approximations in several ways:

(1) Possible iterative schemes for $x_{0}$ are

$$
x_{0}^{(n+1)}=A H x_{0}{ }^{(n)} \text {, }
$$

or

$$
x_{0}{ }^{(n+1)}=A\left(E_{1}{ }^{(n)}-W\right)^{-1}\left(H-E_{0}{ }^{(n)}\right) x_{1}{ }^{(n)},
$$

where in each case the multiplier $A$ is determined from the condition

$$
x_{0}^{(n+1)+} x_{0}^{(n+1)}=1 \text {. }
$$

(2) The obvious iterative scheme for $E_{0}$ is

$$
E_{0}^{(n+1)}=x_{0}^{(n+1)+} H x_{0}^{(n+1)} \text {. }
$$

(3) Possible iterative schemes for $x_{1}$ are 


$$
\left(H-E_{0}^{(n+1)}\right) x_{1}^{(n+1)}=\left(E_{1}^{(n)}-W\right) x_{0}^{(n+1)},
$$

or

$$
\left(H-E_{0}^{(n+1)}\right) x_{1}^{(n+1)}=\left(\langle W\rangle_{0}^{(n+1)}-W\right) x_{0}^{(n+1)},
$$

or

$$
x_{1}^{(n+1)}=\frac{1}{E_{0}^{(n+1)}}\left[H x_{1}^{(n)}+\left(W-{E_{1}}^{(n)}\right) x_{0}^{(n+1)}\right],
$$

or

$$
x_{1}^{(n+1)}=\frac{1}{E_{0}^{(n+1)}}\left[H x_{1}^{(n)}+\left(W-\langle W\rangle_{0}^{(n+1)}\right) x_{0}^{(n+1)}\right] .
$$

(4) Finally, a nonvariational estimate of $E_{1}=x_{0}{ }^{+} W x_{0}$ is

$$
E_{1}{ }^{(n+1)}=\langle W\rangle_{0}{ }^{(n+1)} \equiv x_{0}{ }^{(n+1)+} W x_{0}{ }^{(n+1)},
$$

while the variational estimates we have been discussing may be written

$$
{E_{1}}^{(n+1)}={E_{1}}^{(n)}+x_{1}{ }^{(n+1)+}\left(H-E_{0}{ }^{(n+1)}\right)\left(E_{1}{ }^{(n)}-W\right)^{-1}\left(H-E_{0}{ }^{(n+1)}\right) x_{1}{ }^{(n+1)},
$$

or

$$
\begin{aligned}
{E_{1}}^{(n+1)}= & \langle W\rangle_{0}^{(n+1)} \\
& +x_{1}{ }^{(n+1)+}\left(H-E_{0}^{(n+1)}\right)\left(\langle W\rangle_{0}{ }^{(n+1)}-W\right)^{-1}\left(H-E_{0}{ }^{(n+1)}\right) x_{1}^{(n+1)},
\end{aligned}
$$

or

$$
\begin{aligned}
E_{1}{ }^{(n+1)}=\langle W\rangle^{(n+1)}+x_{0}^{(n+1)+}\left(H-E_{0}{ }^{(n+1)}\right) & x_{1}^{(n+1)} \\
& +x_{1}^{(n+1)+}\left(H-E_{0}{ }^{(n+1)}\right) x_{0}{ }^{(n+1)} .
\end{aligned}
$$

In each of the alternative forms (25), any of the forms (24) may be used for $x_{1}$. We shall consider here several representative overall schemes, which we define as follows: First, (22b) appears to have no advantages over (22a), unless (22a) should not converge. We therefore consider only (22a), and define several approximations to $\langle W\rangle$ in an obvious notation as follows:

$$
\begin{array}{ll}
\langle W\rangle_{0}=E_{1} & (25 \mathrm{a}), \\
\langle W\rangle_{1}=E_{1} & (24 \mathrm{c}, 25 \mathrm{c}), \\
\langle W\rangle_{2}=E_{1} & (24 \mathrm{c}, 25 \mathrm{~d}), \\
\langle W\rangle_{3}=E_{1} & (24 \mathrm{~b}, 25 \mathrm{~d}), \\
\langle W\rangle_{4}=E_{1} & (24 \mathrm{~b}, 25 \mathrm{c}), \\
\langle W\rangle_{5}=E_{1} & (24 \mathrm{a}, 25 \mathrm{~b}), \\
\langle W\rangle_{6}=E_{1} & (24 \mathrm{a}, 25 \mathrm{~d}) .
\end{array}
$$

The forms using (24a), (24b) are at first sight attractive, since these generate directly the product $\left(H-E_{0}\right) x_{1}$, which is all that is required by (25); further, we do not then need to estimate $E_{0}$ separately. However, it is easy to see that they are never a useful sequence. In fact, we have 


$$
\begin{aligned}
& \langle W\rangle_{3}{ }^{(n+1)}=\langle W\rangle_{4}{ }^{(n+1)}=2\langle W\rangle_{0}{ }^{(n)}-\langle W\rangle_{0}{ }^{(n+1)}, \\
& \langle W\rangle_{i}{ }^{(n+1)}=2\langle W\rangle_{i}{ }^{(n)}-\langle W\rangle_{0}{ }^{(n+1)}, \quad i=5,6 .
\end{aligned}
$$

Thus, if the sequence $\langle W\rangle_{0}$ converges, the sequences $\langle W\rangle_{5}$ and $\langle W\rangle_{6}$ in general do not; further, while $\langle W\rangle_{3}$ and $\langle W\rangle_{4}$ converge, they will give at each stage a worse approximation than $\langle W\rangle_{0}$.

An Example. As an example, we consider a simple eigenvalue problem that has been used previously [3]. We take

$$
H=\left(\begin{array}{ccc}
-23 & 11 & 1 \\
11 & -3 & -2 \\
1 & -2 & 1
\end{array}\right), \quad W=\left(\begin{array}{lll}
1 & & \\
& 2 & \\
& & 3
\end{array}\right)
$$

and first approximations $x_{0}{ }^{T(1)}=x_{1}{ }^{T(1)}=(1,0,0)$.

The results for $x_{0}{ }^{(n)}, x_{1}{ }^{(n)}, E_{0}{ }^{(n)}, E_{1}{ }^{(n)}$ are listed in Table I.

TABLE I.

\begin{tabular}{|c|c|c|c|c|c|c|c|c|}
\hline$n$ & $x_{0}^{(n)}$ & $x_{1}^{(n)}$ & $E_{0}^{(n)}$ & $\langle W\rangle_{0}^{(n)}$ & $\langle W\rangle_{1}{ }^{(n)}$ & $\langle W\rangle_{2}{ }^{(n)}$ & $\langle W\rangle_{3}(n)$ & $\mid\langle W\rangle_{5}{ }^{(n)}$ \\
\hline $\begin{array}{l}1 \\
2 \\
3 \\
\infty\end{array}$ & $\begin{array}{c}1,0,0 \\
0.902,-.431,-.039 \\
.913,-.404,-.062 \\
.912,-.406,-.060\end{array}$ & $\begin{array}{c}1,0,0 \\
0.823,-.378,-.033 \\
0.833,-.355,-.051 \\
-\end{array}$ & $\left|\begin{array}{l}-23 \\
-27.93 \\
-27.97 \\
-27.97\end{array}\right|$ & $\begin{array}{l}1 \\
1.188 \\
1.171 \\
1.172\end{array}$ & $\begin{array}{l}1 \\
0.876 \\
1.165 \\
1.172\end{array}$ & $\begin{array}{l}1 \\
1.168 \\
1.172 \\
1.172\end{array}$ & $\begin{array}{l}1 \\
0.812 \\
1.205 \\
1.172\end{array}$ & $\begin{array}{l}1 \\
0.812 \\
0.453 \\
-\end{array}$ \\
\hline
\end{tabular}

Variation iteration results for the matrix element $\langle W\rangle$ defined by equation (28).

The $\langle W\rangle_{i}$ are from different approximate schemes defined in equation (27).

It is seen that, as expected, $\langle W\rangle_{5}$ does not converge at all, while $\langle W\rangle_{3}$ is a very poor approximation. In this example, $\langle W\rangle_{2}$ is a better approximation than $\langle W\rangle_{1}$; this is due to the circumstance that the matrix $W-E_{1}$ is nearly singular.

As expected, the variational estimate $\langle W\rangle_{2}$ is a better approximation than the nonvariational $\langle W\rangle_{0}$. In fact, to the number of figures retained, $\langle W\rangle_{2}^{\left({ }^{(3)}\right.}$ is already equal to $\langle W\rangle^{\infty}$. This example is a testing one since $x_{0}{ }^{(n)}$ converges very rapidly to $x_{0}$; the improvement to be expected in general from the variation principle is greater than shown here.

Acknowledgements. Thanks are due to Professor J. M. Blatt and to Dr. G. H. Derrick for their interest in this work.

University of Sussex

Falmer, Brighton

England

1. L. M. Delves, “A variation principle for arbitrary operators,” Nuclear Phys., v. 41, 1963, pp. 497-503. MR 26 *7317.

2. L. M. Delves, Nuclear Phys., v. 45, 1963, pp. 313-320.

3. D. R. Hartree, Numerical Analysis, Clarendon Press, Oxford, 1952; 2nd ed., 1958, p. 201. MR 14, 690 . 